TRANSACTIONS OF THE

AMERICAN MATHEMATICAL SOCIETY

Volume 361, Number 4, April 2009, Pages 1833-1852

S 0002-9947(08)04532-7

Article electronically published on November 5, 2008

\title{
IMMERSIONS OF SURFACES INTO ASPHERICAL 3-MANIFOLDS
}

\author{
TAHL NOWIK
}

\begin{abstract}
We study finite order invariants of null-homotopic immersions of a closed orientable surface into an aspherical orientable 3-manifold. We give the foundational constructions and classify all order 1 invariants.
\end{abstract}

\section{INTRODUCTION}

Finite order invariants of immersions of a closed orientable surface into $\mathbb{R}^{3}$ have been introduced in [N3, where all order 1 invariants have been classified. Explicit formulae for the majority of order 1 invariants have been given in [N2], [N5]. All higher order invariants have been classified in [N4, and the analogue of all the above for non-orientable surfaces has appeared in [N6]. A first step in the study of finite order invariants of immersions of surfaces into general 3-manifolds appears in N1, where one specific order 1 invariant has been studied.

In the present work we study finite order invariants of null-homotopic immersions of a closed orientable surface $F$ into an aspherical orientable 3 -manifold $M$. We develop the foundations for the study of finite order invariants in our setting, as has been done in $\mathrm{N} 3$ for the case $M=\mathbb{R}^{3}$, and analogous to chord diagrams and the 1-term and 4-term relations in knot theory. We then classify all order 1 invariants. The structure of the set of order 1 invariants will depend on $\pi_{1}(M)$ in an involved way. We will also identify the subset of invariants which are common to all $M$ and give examples of large families of order 1 invariants appearing when $\pi_{1}(M)=\mathbb{Z}^{k}$, which do not appear for $\pi_{1}(M)=0$.

Our procedure for studying finite order invariants is as follows. We try to classify all order $n$ invariants up to order $n-1$ invariants; that is, if $V_{n}$ denotes the group of all invariants of order at most $n$ with values in some Abelian group $\mathbb{G}$, then we would like to understand $V_{n} / V_{n-1}$. This we do in two steps. We first embed $V_{n} / V_{n-1}$ into the group of all functions from some combinatorially constructed set $\mathcal{C}_{n}$ into $\mathbb{G}$. The analogous combinatorially constructed set in knot theory is the set $D_{n}$ of all chord diagrams with $n$ strands. In our case it will be the set of all $n$-tuples of certain symbols involving elements of $\pi_{1}(M)$, up to an equivalence relation involving the right and left action of $\pi_{1}(M)$ on itself. Whereas the set $D_{n}$ of chord diagrams is finite and quite readily understood, our set $\mathcal{C}_{n}$ is infinite, and its complexity depends on the complexity of $\pi_{1}(M)$. A further difference from the setting of knot theory is the matter of co-orientation. Given a singular knot with

Received by the editors February 25, 2007.

2000 Mathematics Subject Classification. Primary 57Mxx, 57R42.

This work was partially supported by the Minerva Foundation.

(C)2008 American Mathematical Society 
$n$ double points, there is a natural way to attach a sign + or - to the two ways for resolving each double point, and this sign is used when an invariant of order $n$ induces a function on $D_{n}$. In our setting there will sometimes be no natural way to attach a sign to each of the two possible resolutions, and at other times there will be no such consistent choice at all; i.e. the stratum in the space of immersions corresponding to certain singularities is one sided. Which strata are one sided will depend on the structure of $\pi_{1}(M)$ (see Examples 3.3 , 3.4). So, as mentioned, the first step for studying finite order invariants is to embed $V_{n} / V_{n-1}$ into $\mathcal{C}_{n}^{*}$, the group of all functions from $\mathcal{C}_{n}$ to $\mathbb{G}$. The second step is to identify precisely what is the image of $V_{n} / V_{n-1}$ in $\mathcal{C}_{n}^{*}$. We will define a certain subgroup $\Delta_{n} \subseteq \mathcal{C}_{n}^{*}$ which contains the image of $V_{n} / V_{n-1}$. The subgroup $\Delta_{n}$ is defined by a certain set of relations that must be satisfied by a function in $\mathcal{C}_{n}^{*}$ in order for it to lie in the image of $V_{n} / V_{n-1}$. These relations are analogous to the 1-term and 4-term relations in knot theory. We will classify all order 1 invariants by showing that the image of $V_{1} / V_{0}$ in $\mathcal{C}_{1}^{*}$ is all $\Delta_{1}$ (Theorem [5.2). This in general does not hold for higher order invariants, as seen in [N4] for the case $M=\mathbb{R}^{3}$, where additional relations appear for higher order invariants.

\section{The Universal COVERING SPACE}

Let $M$ be an aspherical orientable 3-manifold, i.e. $\pi_{n}(M)=0$ for all $n \geq 2$ (a sufficient condition being that $\pi_{2}(M)=\pi_{3}(M)=0$ ). Let $r: \widehat{M} \rightarrow M$ be the universal covering, so $\widehat{M}$ is contractible. For $a \in M$ denote $\pi_{a}=\pi_{1}(M, a)$. In this section we will define a labeling by elements of $\pi_{a}$ of all points in $\widehat{M}$. For each $p \in M$ this labeling will be a bijection between $r^{-1}(p)$ and $\pi_{a}$. We will determine in what way this labeling depends on certain choices and will determine how certain natural actions on $M$ and $r^{-1}(p)$ are represented in terms of this labeling. These labelings by elements of $\pi_{a}$ will be used in Section 3 to construct the symbols mentioned in the Introduction. Namely, given an immersion $i: F \rightarrow M$, the labelings of points of $\widehat{M}$ will induce such a labeling of the points of $F$ via a lift $\hat{i}: F \rightarrow \widehat{M}$. We will use the labels of the points of $F$ participating in a given singularity to construct a symbol characterizing this singularity.

If $\gamma$ is a path in $M$ with initial point $a$ and if $x \in r^{-1}(a)$, then we denote the lift of $\gamma$ to $\widehat{M}$ with initial point $x$ by $\gamma^{x}$. If $p \in M$ is another point and $\gamma$ is a path from $a$ to $p$, then we define a bijection $F_{\gamma}^{x}: \pi_{a} \rightarrow r^{-1}(p)$ by $F_{\gamma}^{x}(\phi)=(\phi * \gamma)^{x}(1)$, where $*$ denotes concatenation from left to right.

We choose $a \in M, x \in r^{-1}(a)$, and for each $p \in M$ we choose a path $\gamma_{p}$ from $a$ to $p$. We then label the points in each $r^{-1}(p)$ by elements of $\pi_{a}$ via the bijection $F_{\gamma_{p}}^{x}$. We now check in what way this labeling depends on our choices. So let $b \in M, y \in r^{-1}(b)$ and for each $p \in M$, let $\delta_{p}$, a path from $b$ to $p$, be another such choice. In order to identify the elements of $\pi_{a}$ with those of $\pi_{b}$ we need to make one additional choice, the dependence on which will be apparent as well. Namely we choose a path $\mu$ from $a$ to $b$ and identify $\pi_{a}$ with $\pi_{b}$ by $\phi \mapsto \bar{\mu} * \phi * \mu$, where $\bar{\mu}$ is the path inverse to $\mu$ (i.e. $\bar{\mu}(t)=\mu(1-t)$ ). Finally, let $s$ be the unique (up to homotopy) path in $M$ from $a$ to $b$ such that $s^{x}(1)=y$. Now for each $p \in M: F_{\delta_{p}}^{y}(\bar{\mu} * \phi * \mu)=\left(\bar{\mu} * \phi * \mu * \delta_{p}\right)^{y}(1)=\left(\bar{\mu} * \phi * \mu * \delta_{p} * \overline{\gamma_{p}} * \gamma_{p}\right)^{s^{x}(1)}(1)=$ $\left(s * \bar{\mu} * \phi * \mu * \delta_{p} * \bar{\gamma}_{p} * \gamma_{p}\right)^{x}(1)=F_{\gamma_{p}}^{x}\left((s * \bar{\mu}) * \phi *\left(\mu * \delta_{p} * \overline{\gamma_{p}}\right)\right)$. So given the identification between $\pi_{a}$ and $\pi_{b}$ determined by $\mu$, the labeling of the points in $r^{-1}(p)$ have 
changed by left multiplication by $s * \bar{\mu}$ and right multiplication by $\mu * \delta_{p} * \overline{\gamma_{p}}$. Note that $s * \bar{\mu}$ depends only on $a, b, x, y, \mu$, and so we get left multiplication by the same element for all points $p$. We summarize this in the following:

Proposition 2.1. The labeling of the points of $r^{-1}(p)$ by elements of $\pi_{a}$ is well defined up to left multiplication by one common element in $\pi_{a}$ for all $p$, and right multiplication by an element in $\pi_{a}$ which may depend on $p$.

Once the dependence on choices has been established, we fix $a, x,\left\{\gamma_{p}\right\}_{p \in M}$ once and for all, and so the labeling of the points in $r^{-1}(p)$ for each $p \in M$ is from now on fixed. Note that this labeling is necessarily not locally constant.

There will be two families of bijections that we will have occasion to use, and we will now see how they are expressed in terms of the labeling. First let $D: \widehat{M} \rightarrow \widehat{M}$ be a deck transformation; then the restriction of $D$ to each $r^{-1}(p)$ is a bijection onto itself. Let $\psi \in \pi_{a}$ be the unique element such that $\psi^{x}(1)=D(x)$. Then for any $\phi \in \pi_{a}$ and any $p \in M, D\left(F_{\gamma_{p}}^{x}(\phi)\right)=D\left(\left(\phi * \gamma_{p}\right)^{x}(1)\right)=\left(\phi * \gamma_{p}\right)^{\psi^{x}(1)}(1)=$ $\left(\psi * \phi * \gamma_{p}\right)^{x}(1)=F_{\gamma_{p}}^{x}(\psi * \phi)$, that is, in terms of the labeling, $D$ is given by common left multiplication by $\psi$. (By common we mean as above that it is the same $\psi$ for all $p$.) Secondly, given $p, q \in M$ let $\delta$ be a path from $p$ to $q$; then $\delta$ defines a bijection $G_{\delta}: r^{-1}(p) \rightarrow r^{-1}(q)$ given by $G_{\delta}(y)=\delta^{y}(1)$. We have for any $\phi \in \pi_{a}, G_{\delta}\left(F_{\gamma_{p}}^{x}(\phi)\right)=G_{\delta}\left(\left(\phi * \gamma_{p}\right)^{x}(1)\right)=\delta^{\left(\phi * \gamma_{p}\right)^{x}(1)}(1)=\left(\phi * \gamma_{p} * \delta\right)^{x}(1)=$ $\left(\phi *\left(\gamma_{p} * \delta * \overline{\gamma_{q}}\right) * \gamma_{q}\right)^{x}(1)=F_{\gamma_{q}}^{x}\left(\phi *\left(\gamma_{p} * \delta * \overline{\gamma_{q}}\right)\right)$; that is, in terms of the labeling, $G_{\delta}$ is given by right multiplication by $\gamma_{p} * \delta * \overline{\gamma_{q}}$.

It is also clear from the above calculations that any common left multiplying element and any right multiplying element can be realized by appropriately choosing $D$ and $\delta$ respectively. We summarize this in the following:

Proposition 2.2. (1) The effect of a deck transformation is a common left multiplication, and any such common left multiplication can be realized by a deck transformation.

(2) The effect of $G_{\delta}$ is right multiplication, and any such right multiplication can be realized by some $\delta$ from $p$ to $q$.

We have noted that our labeling is not locally constant. By Proposition 2.2(2) we see that any such discontinuity is always given by right multiplication.

Denote by $\mathbb{Z}\left[\pi_{a}\right]$ the group ring of $\pi_{a}$ with coefficients in $\mathbb{Z}$. We will in fact not use the full ring structure, but only the left and right actions of $\pi_{a}$ on $\mathbb{Z}\left[\pi_{a}\right]$. For each $p \in M, H_{2}\left(\widehat{M}-r^{-1}(p)\right)$ is a free Abelian group with basis in one-to-one correspondence with $r^{-1}(p)$. This is true by a Mayer-Vietoris sequence, since $\widehat{M}$ is contractible. So via our labeling, for each $p \in M$ we may identify $H_{2}\left(\widehat{M}-r^{-1}(p)\right)$ with $\mathbb{Z}\left[\pi_{a}\right]$.

Any deck transformation induces an automorphism of $H_{2}\left(\widehat{M}-r^{-1}(p)\right)$ for each $p$. It follows from Proposition 2.2(1) that in terms of the identification of $H_{2}\left(\widehat{M}-r^{-1}(p)\right)$ with $\mathbb{Z}\left[\pi_{a}\right]$, this automorphism (of $\mathbb{Z}\left[\pi_{a}\right]$ as an Abelian group) is given by left multiplication by some common $\psi \in \pi_{a}$. Similarly, given $p, q \in M$, a path $\delta$ from $p$ to $q$ naturally induces an isomorphism from $H_{2}\left(\widehat{M}-r^{-1}(p)\right)$ to $H_{2}\left(\widehat{M}-r^{-1}(q)\right)$, and it follows from Proposition 2.2(2) that in terms of the identifications with $\mathbb{Z}\left[\pi_{a}\right]$, this isomorphism is given by right multiplication by some element of $\pi_{a}$. 


\section{Co-oriented AB Equivalences}

In this section we introduce the local singularities of immersions which will be used in the next section to define the notion of finite order invariants. These singularities are the analogue of the double points appearing in the definition of finite order knot invariants. We will then construct our symbols and define an equivalence relation on $n$-tuples of symbols, which will involve both the structure of the singular points and the labelings by elements of $\pi_{a}$ appearing in the previous section. The equivalence classes of $n$-tuples of symbols are analogous to the chord diagrams appearing in knot theory.

Let $M$ be an oriented aspherical 3-manifold, let $F$ be a closed oriented surface, and let $\mathcal{A} \subseteq \operatorname{Imm}(F, M)$ be a regular homotopy class of immersions $i: F \rightarrow M$ which are null-homotopic. A CE point of an immersion $i: F \rightarrow M$ is a point of self intersection of $i$ for which the local stratum in $\operatorname{Imm}(F, M)$ corresponding to the self intersection has codimension one. (The letters $\mathrm{CE}$ are the first and last letters of "co-dimension one".) We distinguish four types of CEs which we name $E, H, T, Q$. In the notation of [HK] they are respectively $A_{0}^{2}\left|A_{1}^{+}, A_{0}^{2}\right| A_{1}^{-}, A_{0}^{3} \mid A_{1}, A_{0}^{4}$. The $E$ and $H$ CEs are the elliptic and hyperbolic tangencies ( $E$ and $H$ standing for elliptic and hyperbolic, respectively). The $T \mathrm{CE}$ appears when a line of intersection is tangent to a third sheet of the surface. Resolving the CE into one of the sides creates two new triple points (so $T$ stands for triple points). The $Q$ singularity is a quadruple point (and $Q$ stands for quadruple). The four types of CEs may be demonstrated by the following local models, where by letting $\lambda$ vary we obtain a 1-parameter family of immersions which is transverse to the given codimension 1 stratum, intersecting it at $\lambda=0$ :

$E: \quad z=0, \quad z=x^{2}+y^{2}+\lambda$.

$H: \quad z=0, \quad z=x^{2}-y^{2}+\lambda$.

$T: \quad z=0, \quad y=0, \quad z=y+x^{2}+\lambda$.

$Q: \quad z=0, \quad y=0, \quad x=0, \quad z=x+y+\lambda$.

See Figure 1, which corresponds to some small $\lambda>0$. If $R$ is one of the above four CE types, then we denote by $|R|$ the number of sheets involved in the given configuration; that is, $|E|=|H|=2,|T|=3,|Q|=4$. Let $I_{n} \subseteq \mathcal{A}$ be the space of all immersions with precisely $n$ CEs; in particular, $I_{0}$ is the space of all stable immersions.

A choice of one of the two sides of the local codimension 1 stratum at a given point of the stratum is represented by the choice of $\lambda<0$ or $\lambda>0$ in the formulae above. We will refer to such a choice as a co-orientation for the configuration of the self intersection. A completely different notion of co-orientation that we will encounter is a chosen side of $i(F)$ in $M$ determined by the orientations of $F$ and $M$. To avoid confusion between the two notions, we use the term "co-orientation" only for the former. For the latter we speak of the "preferred side of $i(F)$ in $M$ ".

For types $E$ and $T$, the configuration of the self intersection at the two sides of the stratum is distinct. Namely, for $\lambda<0$ there is an additional 2-sphere in the image of the immersion, and we permanently choose this side $(\lambda<0)$ as our positive side for the co-orientation. For types $H$ and $Q$, the configuration of the self intersection on the two sides of the strata is the same.

We will now further discuss the symmetries of our four configurations. A symmetry of a $\mathrm{CE}$ configuration is an orientation preserving diffeomorphism from a 


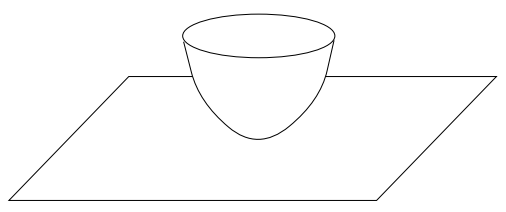

$\mathrm{E}$

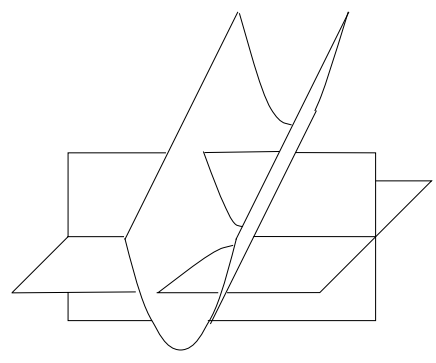

$\mathrm{T}$

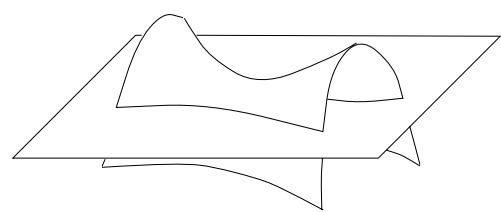

$\mathrm{H}$

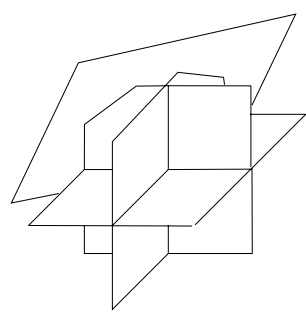

$\mathrm{Q}$

Figure 1. The four CE types

neighborhood of the $\mathrm{CE}$ in $M$ to itself, which maps sheets onto sheets. A symmetry $h$ will be said to preserve the co-orientation of the CE if the image under $h$ of motion into some side of the stratum is again motion into the same side (after identifying each sheet with its image). The comment of the previous paragraph may now be stated as follows: All symmetries of $E, T$ configurations preserve co-orientation, whereas configurations $H, Q$ admit co-orientation reversing symmetries. We further notice that configurations $E, H, T$ admit co-orientation preserving symmetries that realize any given permutation of the sheets, whereas for configuration $Q$, the coorientation preserving symmetries realize only all even permutations of the sheets. These facts may be seen by slightly resolving the CE into one of the sides, and attempting to construct the diffeomorphism $h$ for the resolved configurations.

Given an immersion $i \in I_{n}$, an $n$-co-orientation for $i$ is a choice of co-orientation at each of the $n$ CE points $p_{1}, \ldots, p_{n}$ of $i$. (This is called a temporary co-orientation in [N3, N4, N6.) A proper $n$-co-orientation for $i \in I_{n}$ is an $n$-co-orientation where at each $\mathrm{CE}$ of type $E$ and $T$, the co-orientation chosen is the permanent one mentioned above. (This definition is slightly weaker than that in [N4].)

We now define our symbols mentioned above. Note that whereas for knots it is a singular knot alone that defines a chord diagram, in our setting the symbol will be defined by an immersion $i \in I_{n}$ together with a choice of proper $n$-co-orientation for $i$. So, for immersion $i \in I_{n}$ and proper $n$-co-orientation $\mathfrak{T}$ for $i$, let $p \in M$ be one of its $n$ CE points, and we define $C_{p}(i, \mathfrak{T})$ as the symbol $R_{d}^{g_{1} \epsilon_{1}, \ldots, g_{|R|} \epsilon_{|R|}}$ which 
is composed of:

(1) A symbol $R \in\{E, H, T, Q\}$ which is the configuration of the given $\mathrm{CE}$.

(2) A sequence of elements $g_{1}, \ldots, g_{|R|} \in \pi_{a}$ which is determined as follows: Let $\hat{i}: F \rightarrow \widehat{M}$ be a lift of $i$. There are $|R|$ sheets of $\hat{i}(F)$ passing through points of $r^{-1}(p)$ which in turn are labeled by elements $g_{1}, \ldots, g_{|R|} \in \pi_{a}$ (Section 2). If the $\mathrm{CE}$ is of type $E, H$ or $T$, then the order of the $g_{j} \mathrm{~s}$ is arbitrarily chosen. As to $Q$ configuration, we choose once and for all a rule by which an orientation on a simplex determines an ordering on its four faces, up to an even permutation. Now, we resolve the $\mathrm{CE}$ of type $Q$ positively with respect to $\mathfrak{T}$, i.e. slightly deform $i$ (not $\hat{i}$ ) near $p$, to obtain a stable immersion in a neighborhood of $p$, and write the elements $g_{1}, g_{2}, g_{3}, g_{4}$ in the order determined by the orientation of $M$ restricted to the small simplex created by this resolution. (This ordering convention is related to the symmetry properties discussed above).

(3) To each $g_{j}, 1 \leq j \leq|R|$, there corresponds a sign $\epsilon_{j} \in\{+,-\}$ which is determined as follows: We look at $i: F \rightarrow M$ (not the lift), and resolve the $\mathrm{CE}$ at $p$ according to $\mathfrak{T}$. For types $E, T, Q$ this creates a little sphere in the image of $i$, which bounds a little 3 -cell $V$ in $M$ (for $Q$ this is a simplex, and for $E, T$ recall that $\mathfrak{T}$ is proper). Each $g_{j}$ we have corresponds to a sheet involved in the CE. This sheet may have $V$ on its non-preferred side (determined by the orientations of $F$ and $M$ ), in which case we set $\epsilon_{j}=+$, or otherwise $V$ is on its preferred side, in which case $\epsilon_{j}=-$. For type $H$ the region $V$ is not bounded by the local configuration but may still be defined, e.g. for $\lambda>0$ in the formula for $H$ above, $V$ will be a region consisting of points close to the origin and satisfying $0 \leq z \leq x^{2}-y^{2}+\lambda$. Now the signs $\epsilon_{j}$ are determined by the opposite convention than above. Namely, a sheet having $V$ on its preferred side will have $\epsilon_{j}=+$, and if $V$ is on its non-preferred side we set $\epsilon_{j}=-$. The reason for the opposite convention is to obtain slightly nicer formulae in the end. (It is consistent with the conventions in [N3], chosen there in a similar way for the same reason).

(4) The subscript $d \in \mathbb{Z}\left[\pi_{a}\right]$, which will be called the degree of the CE, is determined as follows: Let $\hat{i}: F \rightarrow \widehat{M}$ be the same lift as used in (2). Wherever $\hat{i}(F)$ passes through a point of $r^{-1}(p)$, we push it slightly into the preferred side of $\hat{i}(F)$ in $\widehat{M}$, obtaining a map $F \rightarrow \widehat{M}-r^{-1}(p)$. This map represents an element of $H_{2}\left(\widehat{M}-r^{-1}(p)\right)$ which we identify with an element $d \in \mathbb{Z}\left[\pi_{a}\right]$ (Section 2).

If $p_{1}, \ldots, p_{n}$ are the $n$ CEs of $i \in I_{n}$, then choose a lift $\hat{i}: F \rightarrow \widehat{M}$ of $i$ and define $C^{\prime}(i, \mathfrak{T})$ to be the $n$-tuple $\left(C_{p_{1}}(i, \mathfrak{T}), \ldots, C_{p_{n}}(i, \mathfrak{T})\right)$, where the same lift $\hat{i}$ is used for all $p_{k}$. Then $C^{\prime}(i, \mathfrak{T}) \in \mathcal{C}_{n}^{\prime}$, where $\mathcal{C}_{n}^{\prime}$ is the set of all $n$-tuples of symbols of the form $R_{d}^{g_{1} \epsilon_{1}, \ldots, g_{|R|} \epsilon_{|R|}}$. In addition to the permanent choices of Section 2. $C^{\prime}$ also depends on the choice of lift $\hat{i}$, and various ordering choices. We thus define an equivalence relation on $\mathcal{C}_{n}^{\prime}$ to be the equivalence relation generated by the following operations:

(1) Any permutation of the $n$ symbols.

(2) For $R=E, H, T$, any permutation of the $|R|$ elements $g_{1} \epsilon_{1}, \ldots, g_{|R|} \epsilon_{|R|}$ (each pair $g_{j} \epsilon_{j}$ goes together).

(3) For $R=Q$, any even permutation of the four elements $g_{1} \epsilon_{1}, \ldots, g_{4} \epsilon_{4}$.

(4) Given $h \in \pi_{a}$, replace each one of the $n$ symbols $R_{d}^{g_{1} \epsilon_{1}, \ldots, g_{|R|} \in|R|}$ of the $n$-tuple by $R_{h d}^{\left(h g_{1}\right) \epsilon_{1}, \ldots,\left(h g_{|R|}\right) \epsilon_{|R|}}$ (the same $h$ for all $n$ symbols).

(5) Given $h \in \pi_{a}$, replace one of the $n$ symbols $R_{d}^{g_{1} \epsilon_{1}, \ldots, g_{|R|} \epsilon_{|R|}}$ by $R_{d h}^{\left(g_{1} h\right) \epsilon_{1}, \ldots,\left(g_{|R|} h\right) \epsilon_{|R|}}$ (it is of course the same $h$ multiplying $g_{j}$ and $d$ on 
the right within one symbol, $h$ being allowed to vary only between the $n$ symbols).

Denote by $\mathcal{C}_{n}$ the set of equivalence classes of $\mathcal{C}_{n}^{\prime}$ under this equivalence relation. Note that because of the common left action in item (4), $\mathcal{C}_{n}$ is not simply the set of unordered $n$-tuples of elements of $\mathcal{C}_{1}$. We now define $C(i, \mathfrak{T}) \in \mathcal{C}_{n}$ to be the equivalence class of $C^{\prime}(i, \mathfrak{T})$. So $C$ is well defined, independent of all choices, including the choice of lift $\hat{i}$ (Proposition 2.2(1)). Denote by $I_{n}^{P}$ the set of all pairs $i, \mathfrak{T}$, where $i \in I_{n}$ and $\mathfrak{T}$ is a proper $n$-co-orientation for $i$. Then $C: I_{n}^{P} \rightarrow \mathcal{C}_{n}$ and we claim:

Lemma 3.1. The map $C: I_{n}^{P} \rightarrow \mathcal{C}_{n}$ is surjective.

Proof. We are given an $n$-tuple $x$ of symbols of the form $R_{d}^{g_{1} \epsilon_{1}, \ldots, g_{|R|} \epsilon_{|R|}}$. Begin with any immersion $i \in \mathcal{A}$ and lift it to $\hat{i}: F \rightarrow \widehat{M}$. Choose points $p_{1}, \ldots, p_{n} \in M$ as locations for the $n$ CEs we will construct. Deform $\hat{i}$ by regular homotopy so that pieces of $F$ will pass the right points of $\widehat{M}$ above each $p_{k}$, to produce the correct labelings appearing as superscripts in the symbols in $x$. Continue the deformation near each such point so that for the projection back to $M$ the right configurations will be created with the right signs and inducing the right ordering in case of CE of type $Q$. Finally, move some other pieces of $F$ across the right points of $\bigcup_{1 \leq k \leq n} r^{-1}\left(p_{k}\right)$ to obtain the correct degrees $d \in \mathbb{Z}\left[\pi_{a}\right]$. The projection back to $M$ gives the desired immersion.

We also note that for types $H, Q$, the relation between the symbols obtained for a given co-orientation of a $\mathrm{CE}$ and that obtained for the opposite co-orientation is as follows: For $H$, all remains the same except for the signs $\epsilon_{j}$ which are all reversed. For $Q$, all signs are reversed, and in addition an odd permutation is performed on $g_{1} \epsilon_{1}, \ldots, g_{4} \epsilon_{4}$. (The degree $d$ remains unchanged since its definition does not involve the co-orientation.) We call the symbol obtained from a symbol of type $H$ or $Q$ in this way the reversed symbol. (Recall that $C_{p}(i, \mathfrak{T})$ is only defined for proper $\mathfrak{T}$, and so for $E, T$ the co-orientation may not be reversed.)

We recall the definition of an $\mathrm{AB}$ equivalence, appearing in [N3: A regular homotopy between two immersions $i, j \in I_{n}$ is called an $\mathrm{AB}$ equivalence if it is alternatingly of type $\mathrm{A}$ and $\mathrm{B}$, where

(1) $J_{t}: F \rightarrow M(0 \leq t \leq 1)$ is of type A if it is of the form $J_{t}=U_{t} \circ i \circ V_{t}$, where $i: F \rightarrow M$ is an immersion and $U_{t}: M \rightarrow M, V_{t}: F \rightarrow F$ are isotopies.

(2) $J_{t}: F \rightarrow M(0 \leq t \leq 1)$ is of type $\mathrm{B}$ if $J_{0} \in I_{n}$ and there are little balls $B_{1}, \ldots, B_{n} \subseteq M$ centered at the $n$ CE points of $J_{0}$ such that $J_{t}$ fixes $U=\left(J_{0}\right)^{-1}\left(\bigcup_{k} B_{k}\right)$ and moves $F-U$ within $M-\bigcup_{k} B_{k}$.

Given two immersions $i, j \in I_{n}$ and $n$-co-orientations $\mathfrak{T}, \mathfrak{T}^{\prime}$ for $i, j$ respectively, we now define co-oriented $\mathrm{AB}$ equivalence, or $\mathrm{CAB}$ equivalence, between $i, \mathfrak{T}$ and $j, \mathfrak{T}^{\prime}$ to be an $\mathrm{AB}$ equivalence between $i$ and $j$ which respects $\mathfrak{T}, \mathfrak{T}^{\prime}$; i.e. if we carry $\mathfrak{T}$ at each $\mathrm{CE}$ of $i$ continuously along the $\mathrm{AB}$ equivalence, then we arrive at $j$ with $n$-co-orientation $\mathfrak{T}^{\prime}$.

The analogue of $\mathrm{AB}$ equivalence in knot theory is the motion of a singular knot which keeps the structure of the double points but allows the knot to cross itself at other regions of the knot. Chord diagrams are precisely those combinatorial objects which classify singular knots up to this motion. In our setting, the elements of $\mathcal{C}_{n}$ 
will be the combinatorial objects which classify properly $n$-co-oriented immersions $i \in I_{n}$ up to $\mathrm{CAB}$ equivalence. Indeed we now prove:

Proposition 3.2. Let $i, j \in I_{n}$ and $\mathfrak{T}, \mathfrak{T}^{\prime} n$-co-orientations for $i, j$ respectively. Then $i, \mathfrak{T}$ and $j, \mathfrak{T}^{\prime}$ are $C A B$ equivalent iff $C(i, \mathfrak{T})=C\left(j, \mathfrak{T}^{\prime}\right)$.

Proof. If $i, \mathfrak{T}$ and $j, \mathfrak{T}^{\prime}$ are CAB equivalent, let $J_{t}(0 \leq t \leq 1)$ be the given $\mathrm{CAB}$ equivalence and let $\mathfrak{T}_{t}(0 \leq t \leq 1)$ be the $n$-co-orientation carried continuously along (so $\mathfrak{T}_{1}=\mathfrak{T}^{\prime}$ ). If a lift $\hat{J}$ of $J$ is used for defining $C^{\prime}\left(J_{t}, \mathfrak{T}_{t}\right)$ at each $t$, and all choices of orderings are also carried along continuously, then by Proposition 2.2(2), $C^{\prime}\left(J_{t}, \mathfrak{T}_{t}\right)$ may only change along the way by right multiplication. We may still have that $J_{1}$ is different than the lift of $j$ used to define $C^{\prime}\left(j, \mathfrak{T}^{\prime}\right)$, in which case we will gain a common left multiplication. The choices of ordering may also be different, but all together we get $C(i, \mathfrak{T})=C\left(j, \mathfrak{T}^{\prime}\right)$.

For the converse, assume $C(i, \mathfrak{T})=C\left(j, \mathfrak{T}^{\prime}\right)$. Since our allowed ordering of superscripts and equivalence of ordering all correspond to the possible symmetries of the configurations, there is an ambient isotopy $U_{t}: M \rightarrow M$ which brings the CEs of $i$ onto the CEs of $j$, such that the $n$-co-orientation $\mathfrak{T}$ carried continuously along coincides with $\mathfrak{T}^{\prime}$, such that the orientations of the corresponding sheets match (since the signs $\epsilon_{j}$ coincide) and such that all labelings in $\pi_{a}$ and degrees in $\mathbb{Z}\left[\pi_{a}\right]$ coincide up to right multiplication by an element in $\pi_{a}$ and common left multiplication by an element in $\pi_{a}$. Since the orientations of corresponding sheets match, we may continue with a regular homotopy of the form $i \circ V_{t}$ for isotopy $V_{t}: F \rightarrow F$ until we have the same discs in $F$ participating in the CEs of $i$ and $j$ and the restriction of $i$ and $j$ to those discs coincides precisely, and we still have matching labeling and degrees up to right and common left multiplication. By Proposition 2.2 any right multiplication can be realized by dragging a CE by an ambient isotopy of $M$ around some loop in $M$ and back to its place to match $j$, and any common left multiplication can be realized by changing the choice of lift. So we perform such ambient isotopies and change of lift until we have that all labelings and degrees coincide precisely. This means that now not only $i, j$ but also their chosen lifts $\hat{i}, \hat{j}$ coincide on the discs participating in the CEs, and that if we slightly deform $i$ and $j$ by pushing each sheet of the CEs slightly into its preferred side, then $\hat{i}, \hat{j}: F \rightarrow \widehat{M}-\bigcup_{1 \leq k \leq n} r^{-1}\left(p_{k}\right)$ represent the same element in $H_{2}\left(\widehat{M}-\bigcup_{1 \leq k \leq n} r^{-1}\left(p_{k}\right)\right)$. (Note that $H_{2}\left(\widehat{M}-\bigcup_{1 \leq k \leq n} r^{-1}\left(p_{k}\right)\right)=$ $\bigoplus_{1 \leq k \leq n} H_{2}\left(\widehat{M}-r^{-1}\left(p_{k}\right)\right)$, where the projections are induced by inclusion.) From this point on we may proceed exactly as in the proof of [N3], Proposition 3.4, where instead of working in $\mathbb{R}^{3}$ with a set of designated points $\left\{p_{1}, \ldots, p_{n}\right\}$, we work in $\widehat{M}$ with a set of designated points $\bigcup_{1 \leq k \leq n} r^{-1}\left(p_{k}\right)$. Note that as in the case of $\mathbb{R}^{3}$, we have $\pi_{2}\left(\widehat{M}-\bigcup_{1 \leq k \leq n} r^{-1}\left(p_{k}\right)\right)=H_{2}\left(\widehat{M}-\bigcup_{1 \leq k \leq n} r^{-1}\left(p_{k}\right)\right)$, and that since $\widehat{M}$ is parallelizable, the obstructions for regular homotopies implied by the Smale-Hirsch Theorem are precisely the same as for $\mathbb{R}^{3}$.

We then compose the obtained regular homotopy with $r$, obtaining the desired $\mathrm{CAB}$ equivalence in $M$.

As already seen in [N3], a CE of type $H$ or $Q$ may be $\mathrm{CAB}$ equivalent to itself with the opposite co-orientation, which means that the stratum corresponding to this $\mathrm{CE}$ in $\operatorname{Imm}(F, M)$ is one sided. From Proposition 3.2 we see that this happens iff the corresponding symbol is equivalent to its reversed symbol. For this to happen 
clearly the number of + and - signs should be equal, as this number is preserved under equivalence. So a one sided stratum may occur only for symbols of the form $H_{d}^{g_{1}+, g_{2}-}$ and $Q_{d}^{g_{1}+, g_{2}+, g_{3}-, g_{4}-}$. If all $g_{j}$ are equal, then we clearly do get a symbol equivalent to its reversed, and so a one sided stratum. In [N3] where $\pi_{1}$ is trivial $\left(M=\mathbb{R}^{3}\right)$, it is thus clear which are the one sided strata. But for a general aspherical manifold this is intricately related to the structure of $\pi_{1}(M)$. In the following two examples we show a case where the stratum for $H_{d}^{g_{1}+, g_{2}-}$ is one sided though $g_{1} \neq g_{2}$, and then a case of an $M$ (with non-trivial $\pi_{1}(M)$ ) where the stratum is one sided only when $g_{1}=g_{2}$.

Example 3.3. Let $M$ be the orientable line bundle over the Klein bottle. Then $M$ is aspherical and $\pi_{1}(M)=\left\langle a, b \mid a^{2} b^{2}=1\right\rangle$. We look at the symbol $H_{0}^{1+, a b-} \in \mathcal{C}_{1}^{\prime}$ (the subscript is the zero element of $\mathbb{Z}\left[\pi_{1}\right]$ ). We have $H_{0}^{1+, a b-}=H_{0}^{a b+, a^{2} b^{2}-}=$ $H_{0}^{a b+, 1-}=H_{0}^{1-, a b+}$. The first equivalence is by left multiplication by $a$ and right multiplication by $b$, the second (which is an actual equality of symbols) is by the relations in $\pi_{1}$, and the third by permuting the superscripts. So the symbol $H_{0}^{1+, a b-}$ is equivalent to its reversed symbol and so the $H_{0}^{1+, a b-}$ stratum is one sided, though $1 \neq a b$.

Example 3.4. Let $M=S^{1} \times \mathbb{R}^{2}$. Let $x$ be a generator of $\pi_{1}(M)$ and say $k<r$. Then $H_{0}^{x^{k}+x^{r}-} \in \mathcal{C}_{1}^{\prime}$ is not equivalent to the reversed symbol $H_{0}^{x^{k}-, x^{r}+}$ since the property of having the + sign attached to the smaller power of $x$ will be preserved under any left or right multiplication by element $x^{s} \in \pi_{1}(M)$.

\section{Finite ORDER InVARIANTS}

In this section, we first define the notion of finite order invariant. We then show how an invariant of order $n$ with values in an Abelian group $\mathbb{G}$ induces a function from $\mathcal{C}_{n}$ to $\mathbb{G}$. Finally we will find a family of relations on such functions which must be satisfied in order for a function to be induced by some order $n$ invariant.

Given an $n$-co-orientation $\mathfrak{T}$ for $i \in I_{n}$ and a subset $A \subseteq\left\{p_{1}, \ldots, p_{n}\right\}$, we define $i_{\mathfrak{T}, A} \in I_{0}$ to be the immersion obtained from $i$ by resolving all CEs of $i$ at points of $A$ into the positive side with respect to $\mathfrak{T}$, and all CEs not in $A$ into the negative side. Now let $\mathbb{G}$ be any Abelian group and let $f: I_{0} \rightarrow \mathbb{G}$ be an invariant; i.e. a function which is constant on each connected component of $I_{0}$. Given $i \in I_{n}$ and an $n$-co-orientation $\mathfrak{T}$ for $i, f^{\mathfrak{T}}(i)$ is defined as follows:

$$
f^{\mathfrak{T}}(i)=\sum_{A \subseteq\left\{p_{1}, \ldots, p_{n}\right\}}(-1)^{n-|A|} f\left(i_{\mathfrak{T}, A}\right),
$$

where $|A|$ is the number of elements in $A$. If $\mathfrak{T}, \mathfrak{T}^{\prime}$ are two $n$-co-orientations for the same immersion $i$, then $f^{\mathfrak{T}}(i)= \pm f^{\mathfrak{T}^{\prime}}(i)$, and so having $f^{\mathfrak{T}}(i)=0$ is independent of the $n$-co-orientation $\mathfrak{T}$. An invariant $f: I_{0} \rightarrow \mathbb{G}$ is called of finite order if there is an $n$ such that $f^{\mathfrak{T}}(i)=0$ for all $i \in I_{n+1}$. The minimal such $n$ is called the order of $f$. The group of all invariants on $I_{0}$ of order at most $n$ is denoted $V_{n}=V_{n}(\mathbb{G})$.

Let $\mathcal{C}_{n}^{*}$ denote the group of all functions from $\mathcal{C}_{n}$ to $\mathbb{G}$. We will now embed $V_{n} / V_{n-1}$ into $\mathcal{C}_{n}^{*}$. An invariant $f \in V_{n}$ induces a function $\mu_{n}(f): \mathcal{C}_{n} \rightarrow \mathbb{G}$ as follows: Given $x \in \mathcal{C}_{n}$ take $(i, \mathfrak{T}) \in I_{n}^{P}$ with $C(i, \mathfrak{T})=x$. Such $i, \mathfrak{T}$ exists by Lemma 3.1. Now define $\mu_{n}(f)(x)=f^{\mathfrak{T}}(i)$. This is well defined since if $j, \mathfrak{T}^{\prime}$ also has $C\left(j, \mathfrak{T}^{\prime}\right)=x$, then by Proposition $3.2, i, \mathfrak{T}$ and $j, \mathfrak{T}^{\prime}$ are $\mathrm{CAB}$ equivalent, from which it follows 
that $f^{\mathfrak{T}}(i)=f^{\mathfrak{T}^{\prime}}(j)$, the argument being the same as in [N3, Proposition 3.8. It is easy to see that the map $f \mapsto \mu_{n}(f)$ is a group homomorphism, and by definition of the order of an invariant its kernel is precisely $V_{n-1}$, so it induces an injection $\mu_{n}: V_{n} / V_{n-1} \rightarrow \mathcal{C}_{n}^{*}$. This injection was the purpose for defining $\mathcal{C}_{n}$ and the notion of $\mathrm{CAB}$ equivalence. Classifying all order $n$ invariants up to order $n-1$ invariants is now the same as determining the image of this injection. The rest of this section is dedicated to defining a certain subgroup $\Delta_{n} \subseteq \mathcal{C}_{n}^{*}$ which contains the image of $\mu_{n}$. It will be defined by a set of relations to be satisfied by functions in $\mathcal{C}_{n}^{*}$.

The first two families of relations are obtained as follows. Let $i \in I_{n}$ and let $\mathfrak{T}, \mathfrak{T}^{\prime}$ be two proper $n$-co-orientations for $i$ which differ at precisely one CE, necessarily of type $H$ or $Q$, and located say at $p \in M$. On the one hand, from the definition of $f^{\mathfrak{T}}(i)$, we have for any $f \in V_{n}: f^{\mathfrak{T}}(i)=-f^{\mathfrak{T}^{\prime}}(i)$. On the other hand, we have already noticed the relation between $C_{p}(i, \mathfrak{T})$ and $C_{p}\left(i, \mathfrak{T}^{\prime}\right)$ in such a case (paragraph following the proof of Lemma 3.1). We thus get equations that must be satisfied by any $g \in \mathcal{C}_{n}^{*}$ in order for it to lie in the image of $\mu_{n}$. Namely, if $Z_{2}, \ldots, Z_{n}$ are any $n-1$ symbols, then for any symbol $H_{d}^{g_{1} \epsilon_{1}, g_{2} \epsilon_{2}}, g$ must satisfy $g\left(H_{d}^{g_{1} \epsilon_{1}, g_{2} \epsilon_{2}}, Z_{2}, \ldots, Z_{n}\right)=-g\left(H_{d}^{g_{1} \hat{\epsilon}_{1}, g_{2} \hat{\epsilon}_{2}}, Z_{2}, \ldots, Z_{n}\right)$, where $\hat{\epsilon}_{j}$ denotes the sign opposite to $\epsilon_{j}$ and where by $g\left(Z_{1}, \ldots, Z_{n}\right)$ we mean the value of $g$ on the equivalence class of the $n$-tuple $\left(Z_{1}, \ldots, Z_{n}\right)$. We will write such an equation in the short form $H_{d}^{g_{1} \epsilon_{1}, g_{2} \epsilon_{2}}=-H_{d}^{g_{1} \hat{\epsilon}_{1}, g_{2} \hat{\epsilon}_{2}}$. For $Q$ configuration we similarly get $Q_{d}^{g_{1} \epsilon_{1}, g_{2} \epsilon_{2}, g_{3} \epsilon_{3}, g_{4} \epsilon_{4}}=$ $-Q_{d}^{g_{2} \hat{\epsilon}_{2}, g_{1} \hat{\epsilon}_{1}, g_{3} \hat{\epsilon}_{3}, g_{4} \hat{\epsilon}_{4}}$ (note the transposition $1 \leftrightarrow 2$ ). Also note that in the above two equations, the operation which produces the $n$-tuple of symbols on the right from the $n$-tuple of symbols on the left is indeed well defined on the classes in $\mathcal{C}_{n}$.

In addition to the above two families of equations, we will now find six more such families by observing small loops in the space of immersions going around co-dimension 2 strata. Let $i \in \mathcal{A}$ be an immersion with a self intersection of local codimension 2 at $p$ and $n-1$ additional self intersections of local codimension 1 (i.e. CEs) at $p_{1}, \ldots, p_{n-1}$. We look at a 2 -parameter family of immersions which moves $F$ only in a neighborhood of $p$, such that the immersion $i$ corresponds to parameters $(0,0)$ and such that this 2-parameter family is transverse to the local codimension 2 stratum at $i$. In this 2-parameter family of immersions we look at a loop which encircles the point of intersection with the codimension 2 strata; i.e. a circle around the origin in the parameter plane. This circle crosses the local codimension 1 strata some $r$ times. Between each of the two intersections we have an immersion in $I_{n-1}$ with the same $n-1$ CEs at $p_{1}, \ldots, p_{n-1}$. At each intersection with the local codimension 1 strata, an $n$th $\mathrm{CE}$ is added, obtaining an immersion in $I_{n}$. Let $i_{1}, \ldots, i_{r}$ be the $r$ immersions in $I_{n}$ so obtained. For each $1 \leq k \leq r$, choose a proper $n$-co-orientation $\mathfrak{T}_{k}$ for $i_{k}$, such that the co-orientation chosen for each $p_{j}$, $1 \leq j \leq n-1$, is the same in all $\mathfrak{T}_{1}, \ldots, \mathfrak{T}_{r}$. Let $e_{k}, k=1, \ldots, r$, be + or - according to whether we are passing the $n$th CE of $i_{k}$ in the direction of its co-orientation determined by $\mathfrak{T}_{k}$ or in the opposite direction, respectively. For an invariant $f$, it is easy to show that the following equation holds: $\sum_{k=1}^{r} e_{k} f^{\mathfrak{T}_{k}}\left(i_{k}\right)=0$. Looking at $\mu_{n}: V_{n} / V_{n-1} \rightarrow \mathcal{C}_{n}^{*}$ we thus obtain additional equations that must be satisfied by a function in $\mathcal{C}_{n}^{*}$ in order for it to lie in the image of $\mu_{n}$. We will now find all equations on $\mathcal{C}_{n}^{*}$ obtained in this way. As above, the equations will be written in short form as equations on the symbols. We may assume (by moving the codimension 2 singular point $p$ if necessary) that there is a neighborhood $U$ of $p$ such that the labeling by elements of $\pi_{a}$ is locally constant in $r^{-1}(U)$ and such that all motion involved in 
our 2-parameter family of immersions takes place inside $U$. Therefore, we will be able to follow the labelings during our loop of immersions.

The local codimension 2 strata may be divided into six types which we name after the types of CEs appearing in a 2-parameter family of immersions, transverse to the given stratum: $E H, T T, E T, H T, T Q, Q Q$. In the notation of [HK they are respectively: $A_{0}^{2}\left|A_{2}, A_{0}^{3}\right| A_{2},\left(A_{0}^{2} \mid A_{1}^{+}\right)\left(A_{0}\right),\left(A_{0}^{2} \mid A_{1}^{-}\right)\left(A_{0}\right),\left(A_{0}^{3} \mid A_{1}\right)\left(A_{0}\right), A_{0}^{5}$.

For each of the first five types we give the following:

(1) Formula for a local representative.

(2) Sketch of the configuration for some value $\left(\lambda_{1}, \lambda_{2}\right)$ of the parameters.

(3) Diagram of the 2 dimensional parameter space, where the intersection with the codimension 1 strata is depicted, including some choice of proper coorientations (this is called a bifurcation diagram).

(4) The relation arising, using the given proper co-orientation.

The symbol $\cdots$ appearing in a diagram or equation represents a string of $g_{j} \epsilon_{j} \mathrm{~s}$ of the appropriate length (the same string for all appearances of $\cdots$ within the same diagram or equation). For these five types, the bifurcation diagram is obtained from the sketch and formula in a straightforward manner. We then go on to type $Q Q$; it requires special analysis which will be done in detail.
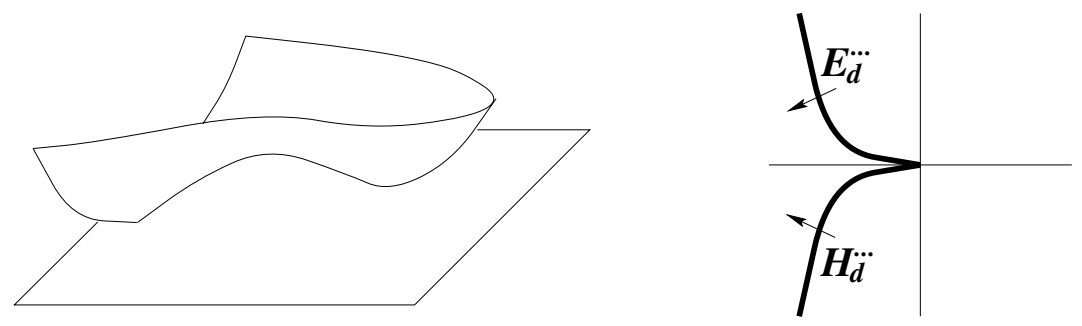

Figure 2. $E H$ configuration

$E H: \quad z=0, \quad z=y^{2}+x^{3}+\lambda_{1} x+\lambda_{2}$.

$$
0=E_{d}^{\cdots}-H_{d}^{\cdots}
$$
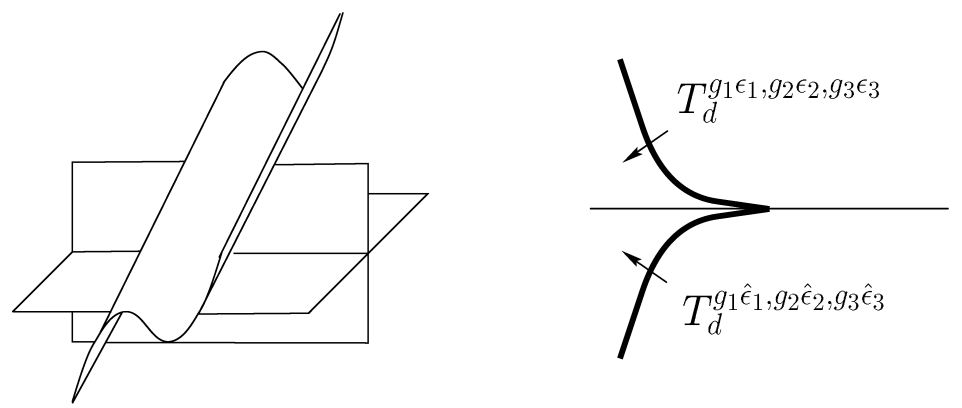

Figure 3. TT configuration

TT: $\quad z=0, \quad y=0, \quad z=y+x^{3}+\lambda_{1} x+\lambda_{2}$.

$$
0=T_{d}^{g_{1} \epsilon_{1}, g_{2} \epsilon_{2}, g_{3} \epsilon_{3}}-T_{d}^{g_{1} \hat{\epsilon}_{1}, g_{2} \hat{\epsilon}_{2}, g_{3} \hat{\epsilon}_{3}}
$$



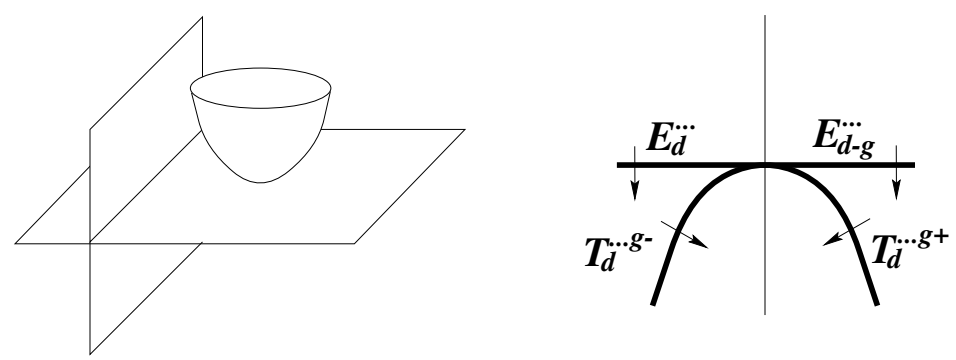

FiguRE 4. ET configuration

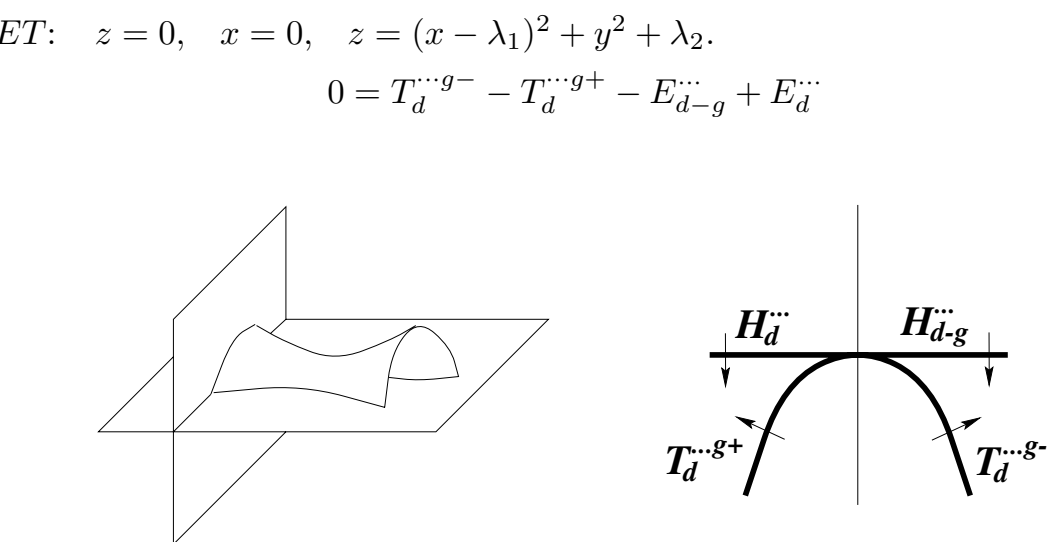

FiguRE 5. HT configuration

$H T: \quad z=0, \quad x=0, \quad z=\left(x-\lambda_{1}\right)^{2}-y^{2}+\lambda_{2}$.

(4)

$$
0=-T_{d}^{\cdots g+}+T_{d}^{\cdots g-}-H_{d-g}^{\cdots}+H_{d}^{\cdots}
$$
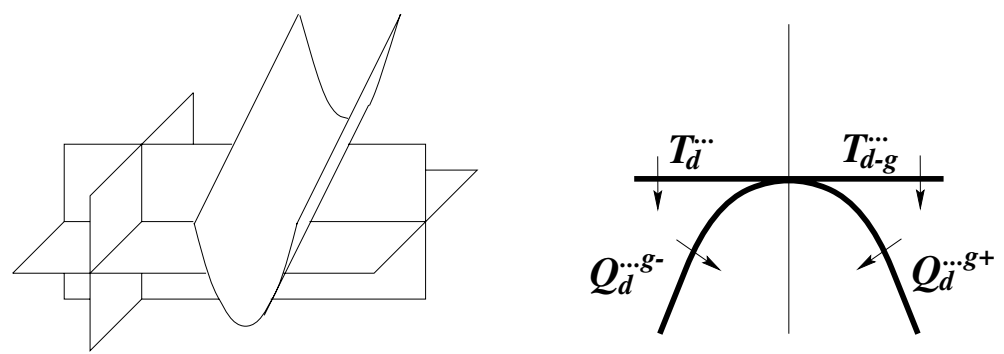

Figure 6. TQ configuration

$T Q: \quad z=0, \quad y=0, \quad x=0, \quad z=y+\left(x-\lambda_{1}\right)^{2}+\lambda_{2}$.

$$
0=Q_{d}^{\cdots g-}-Q_{d}^{\cdots g+}-T_{d-g}^{\cdots}+T_{d}^{\cdots}
$$

$Q Q$ : This configuration is a quintuple point; i.e. five sheets passing through a point, any three of which are in general position. We model this by five planes passing through a point in $\mathbb{R}^{3}$. A 2-parameter family may be constructed by fixing 
three of the planes and allowing the other two planes to move parallel to themselves. A loop in the shape of a rectangle around the origin of the parameter plane may be constructed as follows: The three fixed planes $P_{1}, P_{2}, P_{3}$ intersect in a triple point $x$. Start with the plane $P_{4}$ very close to $x$, and $P_{5}$ somewhat further away. For the first edge of the rectangle, move $P_{4}$ across $x$ passing a first quadruple point. In this process a little simplex $S^{-}$has vanished and a new little simplex $S^{+}$has been created from the same four planes $P_{1}, \ldots, P_{4}$. For the second edge of the rectangle, move $P_{5}$ across the whole of $S^{+}$, passing four quadruple points on the way, when crossing the four triple points of $S^{+}$. For the third edge move $P_{4}$ back to its original place, thus crossing $x$ again, in the opposite direction, recreating $S^{-}$in place of $S^{+}$, this being the sixth quadruple point. Finally move $P_{5}$ back to place, across $S^{-}$, adding four more quadruple points, and so completing a total of ten quadruple points. We co-orient each of these ten quadruple points according to the direction we are passing it along this loop, and so each of the ten terms in the equation that we are producing will have a + sign in front of it.

We will call the first and third edges of the rectangle described above "short edges" (since they pass only one quadruple point) and the second and fourth edges "long edges". We will first find the relation between the symbols of two consecutive quadruple points along a long edge, and then see that the same relation holds when passing to a short edge, and back. So let $p, q$ be two vertices of the little simplex, which $P_{5}$ passes consecutively. Let $e$ be the edge of the simplex connecting $p, q$. By affine transformation of $\mathbb{R}^{3}$ we may assume that $p=(0,0,-1), q=(0,0,1)$, that the two planes intersecting to create the edge $e$ are the planes $\{x=0\}$ and $\{y=0\}$, and that the plane $P_{5}$ which is in motion, crossing $p$ and then $q$, is the plane $\{z=t\}$, where $t$ increases in the range $-1-r<t<1+r$ for some small $r$. For concreteness we look at the time $t=0$, and so $P_{5}=\{z=0\}$. As to the remaining two planes, one must pass $p$, and the other must pass $q$. They cannot be vertical planes (since we already have two vertical planes), and so they are given by $\{z=-1+a x+b y\}$ and $\{z=1+c x+d y\}$. The coefficients $a, b$ must both be non-zero since if say $b=0$, then we get three planes $\{z=-1+a x\},\{z=0\}$, $\{x=0\}$ which are not in general position (when moved parallel to themselves to have a common point). Similarly $c, d$ are non-zero. By rotating the configuration around the $z$ axis we may assume $a, b>0$. We claim that we must then also get $c, d>0$. Indeed, in order that $P_{5}$ will pass $p$ and $q$ consecutively, it must be that the other two vertices of the simplex lie outside the region $-1 \leq z \leq 1$ of $\mathbb{R}^{3}$. These two points are $s_{1}=\{z=-1+a x+b y\} \cap\{z=1+c x+d y\} \cap\{x=0\}$ and $s_{2}=\{z=$ $-1+a x+b y\} \cap\{z=1+c x+d y\} \cap\{y=0\}$. The $z$ component of $s_{1}$ is $\frac{b+d}{b-d}$, and this is outside the interval $[-1,1]$ iff $d>0$. (If $b=d$, then there is no intersection, which implies that the three planes are not in general position.) In the same way $c>0$, using $s_{2}$. Therefore, the simplex $S_{p}^{+}$created from the passage of $P_{5}$ across $p$ (bounded by the planes $\{x=0\},\{y=0\},\{z=0\},\{z=-1+a x+b y\}$ ) lies in the octant $x \geq 0, y \geq 0, z \leq 0$, whereas the simplex $S_{q}^{-}$that is about to vanish when $P_{5}$ arrives at $q$ (bounded by the planes $\{x=0\},\{y=0\},\{z=0\},\{z=1+c x+d y\}$ ) lies in the octant $x \leq 0, y \leq 0, z \geq 0$, which is the exact opposite octant. Therefore the sign $\epsilon_{j}$ with which each of the common planes $\{x=0\},\{y=0\},\{z=0\}$ appears in $S_{p}^{+}$and $S_{q}^{-}$is the opposite sign. But since we have co-oriented all quadruple points according to our direction of motion, the simplex $S_{q}^{-}$is not the one with which we determine the signs for the symbol $Z_{q}$ of the quadruple point at $q$, but rather the 
simplex $S_{q}^{+}$which will be created after we cross $q$. As we have already noticed, the signs of the faces for $S_{q}^{+}$are all opposite to the corresponding signs for $S_{q}^{-}$, and so finally the common planes with $S_{p}^{+}$will have the same sign as in $S_{p}^{+}$.

We apply a similar two step argument to determine the relation between the orderings of the faces in the two symbols $Z_{p}, Z_{q}$. We note that if some ordering of the faces of $S_{p}^{+}$is consistent with the orientation of $S_{p}^{+}$(restricted from $\mathbb{R}^{3}$ ), then the ordering for the faces of $S_{q}^{-}$obtained by simply replacing the plane $\{z=$ $-1+a x+b y\}$ with the plane $\{z=1+c x+d y\}$ is inconsistent with the orientation of $S_{q}^{-}$. Therefore, this same ordering of these same four planes, now considered as the faces of $S_{q}^{+}$, is consistent with the orientation of $S_{q}^{+}$. So for the superscripts of two consecutive symbols we finally get that the $g_{j} \epsilon_{j}$ corresponding to the plane which appears only in the first quadruple point and not the second (the plane $\{z=-1+a x+b y\}$ in our case) should be dropped, and exactly in its place should be written the $g_{j} \epsilon_{j}$ corresponding to the new plane which participates in the second quadruple point but not in the first (the plane $\{z=1+c x+d y\}$ in our case). The three $g_{j} \epsilon_{j}$ s corresponding to the three common planes (the planes $\{x=0\},\{y=0\},\{z= \pm 1\}$ in our case) must remain in their place, and with the signs $\epsilon_{j}$ unchanged.

We now show that when passing from the last quadruple point of a long edge to the quadruple point of the next short edge, or from that to the first quadruple point of the next long edge, then the same relation holds. Indeed, looking say at the first and second quadruple point in our rectangle described above, let $y$ be the triple point of $S^{+}$which $P_{5}$ passes first along the long edge, for the occurrence of the second quadruple point. Let $P$ be one of the planes $P_{1}, \ldots, P_{4}$ such that if $P$ is moved in the direction such that $S^{+}$increases in size, then the point $y$ will move toward $P_{5}$ and eventually cross it. So we can start from the same initial position as before, but instead of moving $P_{4}$ and then $P_{5}$, we move only plane $P$, such that $S^{-}$ will vanish, passing the first quadruple point, $S^{+}$is created and increases in size until $y$ crosses $P_{5}$, thus passing the second quadruple point. So we have obtained the same two quadruple points, where now four of the planes are fixed and only the plane $P$ is in motion. But this is precisely the motion analyzed above to obtain the relation between two consecutive quadruple points along a long edge.

Finally it remains to determine the degrees $d \in \mathbb{Z}\left[\pi_{a}\right]$ of the ten symbols. We do this again by analyzing the relation between the degrees of two consecutive quadruple points. Following the intersection point between $\{x=0\},\{y=0\},\{z=$ $t\}$ as it continuously moves up from $p$ to $q$, we see that there is no change in the degree due to these three planes. (Recall that the labeling is locally constant above the region of interest.) There may, on the other hand, be a change due to the planes $P_{i}=\{z=-1+a x+b y\}$ and $P_{j}=\{z=1+c x+d y\}$. If the preferred side of $P_{i}$ is facing upward, which happens iff the corresponding sign $\epsilon_{i}$ appearing in the symbol $Z_{p}$ for the first quadruple point is - , then when computing the degree $d_{p}$ for the first quadruple point, $P_{i}$ is slightly pushed upward so as to pass above the point $p$. But as $P_{5}$ moves upward to $q, P_{i}$ does not participate in the second quadruple point, and remains below the point $q$, which is where the degree is now computed. So in this case there is a change of $-g_{i}$ to the degree due to the plane $P_{i}$. If on the other hand the plane $P_{i}$ is facing downward, which happens iff the sign $\epsilon_{i}$ appearing in $Z_{p}$ is + , then the plane $P_{i}$ is pushed downward when $d_{p}$ is computed, and again it will remain below the point $q$ when the degree of the second quadruple point is 
computed, so there will be no change in the degree due to $P_{i}$. The two cases may be written in one formula as follows. For sign $\epsilon \in\{+,-\}$, define

$$
|\epsilon|= \begin{cases}1 & \text { if } \epsilon=+ \\ 0 & \text { if } \epsilon=-;\end{cases}
$$

then the change in degree between the first and second quadruple point, due to the plane $P_{i}$, is $-\left|\hat{\epsilon}_{i}\right| g_{i}$. A similar analysis as to the effect of the plane $P_{j}$ on the degree will give $+\left|\epsilon_{j}\right| g_{j}$. Together we get that if $P_{j}$ is the plane missing from the first quadruple point, $P_{i}$ appears there with sign $\epsilon_{i}, P_{i}$ is missing from the second quadruple point, and $P_{j}$ appears there with sign $\epsilon_{j}$, then the change in the degree between the first and second quadruple points is $+\left|\epsilon_{j}\right| g_{j}-\left|\hat{\epsilon}_{i}\right| g_{i}$.

Looking at the circle (or rectangle) of ten quadruple points, we see that the same 4-tuple of planes participates in a quadruple point twice, and with opposite co-orientation, and so the ordering of the corresponding $g_{i} \epsilon_{i}$ at the two times differ by an odd permutation. They occur at the precise opposite timing along the circle, i.e. five places apart, and so the ordering of quadruple points along the circle is that all five possible 4-tuples appear one after the other, and then they appear again in the same order, to complete the cycle of ten quadruple points. So given a quadruple point, either it or the matching quadruple point on the opposite side of the circle have the property that the ordering of superscripts may be chosen as $g_{1} \epsilon_{1}, \ldots, g_{4} \epsilon_{4}$ so that in the next quadruple point the plane corresponding to $g_{1}$ will be missing, in the following quadruple point the plane corresponding to $g_{2}$ will be missing, and so on. Finally, since the two matching quadruple points (i.e. those with the same 4-tuple of planes) occur with opposite co-orientation, the sign attached to each $g_{i}$ will be opposite (i.e. $g_{i} \epsilon_{i}$ is replaced by $g_{i} \hat{\epsilon}_{i}$ ). Since we have seen that if a plane is common to two consecutive quadruple points, it appears in their superscripts with the same sign, and since a given plane will be missing exactly once between its appearance in a given quadruple point and the occurrence of the matching quadruple point, it follows that whenever a plane is missing from a quadruple point, then it appears in the following quadruple point with opposite sign than it does in the previous one.

To write our final formula, let us choose the first quadruple point and ordering $g_{1} \epsilon_{1}, \ldots, g_{4} \epsilon_{4}$ as mentioned above, and we may write its degree as $d-\left|\epsilon_{5}\right| g_{5}$, where $g_{5}$ corresponds to the plane missing from the first quadruple point and $\epsilon_{5}$ is the sign with which it will appear in the second quadruple point. The first symbol is thus $Q_{d-\left|\epsilon_{5}\right| g_{5}}^{g_{1} \epsilon_{1}, g_{2} \epsilon_{2}, g_{3} \epsilon_{3}, g_{4} \epsilon_{4}}$, and the combination of all analysis above produces the following equation:

(6)

$$
\begin{aligned}
& 0=Q_{d-\left|\epsilon_{5}\right| g_{5}, g_{3} \epsilon_{3}, g_{4} \epsilon_{4}}^{g_{1} \epsilon_{1} g_{1} \epsilon_{2}}+Q_{d-\left|\hat{\epsilon}_{1}\right| g_{1}}^{g_{5} \epsilon_{5}, g_{2}, g_{3} \epsilon_{3}, g_{4} \epsilon_{4}}+Q_{d-\left|\hat{\epsilon}_{2}\right| g_{2}}^{g_{5} \epsilon_{5}, g_{1} \hat{\epsilon}_{1}, g_{3} \epsilon_{3}, g_{4} \epsilon_{4}}+Q_{d-\left|\hat{\epsilon}_{3}\right| g_{3}}^{g_{5} \epsilon_{5}, \hat{\epsilon}_{1}, g_{2} \hat{\epsilon}_{2}, g_{4} \epsilon_{4}} \\
& +Q_{d-\left|\hat{\epsilon}_{4}\right| g_{4}}^{g_{5} \epsilon_{5}, g_{1} \hat{\epsilon}_{1}, g_{2} \hat{\epsilon}_{2}, g_{3} \hat{\epsilon}_{3}}+Q_{d-\left|\hat{\epsilon}_{5}\right| g_{5}}^{g_{4} \hat{\epsilon}_{4}, g_{1} \hat{\epsilon}_{1}, g_{2} \hat{\epsilon}_{2}, g_{3} \hat{\epsilon}_{3}}+Q_{d-\left|\epsilon_{1}\right| g_{1}}^{g_{4} \hat{\epsilon}_{4}, g_{5} \hat{\epsilon}_{5}, g_{2} \hat{\epsilon}_{2}, g_{3} \hat{\epsilon}_{3}}+Q_{d-\left|\epsilon_{2}\right| g_{2}}^{g_{4} \hat{\epsilon}_{4}, g_{5} \hat{\epsilon}_{5}, g_{1} \epsilon_{1}, g_{3} \hat{\epsilon}_{3}} \\
& +Q_{d-\left|\epsilon_{3}\right| g_{3}}^{g_{4} \hat{\epsilon}_{4}, g_{5} \hat{\epsilon}_{5}, g_{1} \epsilon_{1}, g_{2} \epsilon_{2}}+Q_{d-\left|\epsilon_{4}\right| g_{4}}^{g_{3} \epsilon_{3}, g_{5} \hat{\epsilon}_{5}, g_{1} \epsilon_{1}, g_{2} \epsilon_{2}} .
\end{aligned}
$$

We summarize what we have achieved in the following definition and proposition:

Definition 4.1. Let $\Delta_{n}=\Delta_{n}(\mathbb{G}) \subseteq \mathcal{C}_{n}^{*}$ be the subgroup consisting of all functions $g \in \mathcal{C}_{n}^{*}$ which satisfy equations (1) - (6) together with our first two equations $H_{d}^{g_{1} \epsilon_{1}, g_{2} \epsilon_{2}}=-H_{d}^{g_{1} \hat{\epsilon}_{1}, g_{2} \hat{\epsilon}_{2}}$ and $Q_{d}^{g_{1} \epsilon_{1}, g_{2} \epsilon_{2}, g_{3} \epsilon_{3}, g_{4} \epsilon_{4}}=-Q_{d}^{g_{2} \hat{\epsilon}_{2}, g_{1} \hat{\epsilon}_{1}, g_{3} \hat{\epsilon}_{3}, g_{4} \hat{\epsilon}_{4}}$. 
Proposition 4.2. The image of the injection $\mu_{n}: V_{n} / V_{n-1} \rightarrow \mathcal{C}_{n}^{*}$ is contained in $\Delta_{n}$.

Finding the precise image of $\mu_{n}$ for all $n$ (as has been done for the case $M=\mathbb{R}^{3}$ in [N3, N4]) would give a full classification of all finite order invariants. In the next section we will show that the image of $\mu_{1}$ is all $\Delta_{1}$, by this classifying all order one invariants.

\section{ORDER ONE INVARIANTS}

We define a "universal" Abelian group $\mathbb{G}_{U}$ by the Abelian group presentation which takes as generators the elements of $\mathcal{C}_{1}$ and as relations the set of all equations used above to define $\Delta_{n}$ (and in particular $\Delta_{1}$ ). Note that for defining $\Delta_{1}$, an expression such as $H_{d}^{g_{1} \epsilon_{1}, g_{2} \epsilon_{2}}=-H_{d}^{g_{1} \hat{\epsilon}_{1}, g_{2} \hat{\epsilon}_{2}}$ is merely a short form for writing $g\left(H_{d}^{g_{1} \epsilon_{1}, g_{2} \epsilon_{2}}\right)=-g\left(H_{d}^{g_{1} \hat{\epsilon}_{1}, g_{2} \hat{\epsilon}_{2}}\right)$, this being a condition on an element $g \in \mathcal{C}_{1}^{*}$ for being included in the subgroup $\Delta_{1}$, whereas, in the definition of $\mathbb{G}_{U}$, $H_{d}^{g_{1} \epsilon_{1}, g_{2} \epsilon_{2}}=-H_{d}^{g_{1} \hat{\epsilon}_{1}, g_{2} \hat{\epsilon}_{2}}$ is an actual relation in the presentation of $\mathbb{G}_{U}$ by generators and relations. We define the universal element $g^{U} \in \Delta_{1}\left(\mathbb{G}_{U}\right)$ to be the function that assigns to each element in $\mathcal{C}_{1}$ the generator corresponding to it in $\mathbb{G}_{U}$. By definition of $\mathbb{G}_{U}$, indeed $g^{U} \in \Delta_{1}\left(\mathbb{G}_{U}\right)$. We will now establish the existence of an order 1 invariant $f^{U}: I_{0} \rightarrow \mathbb{G}_{U}$ satisfying $\mu_{1}\left(f^{U}\right)=g^{U}$. This will prove our desired result, that for any Abelian group $\mathbb{G}, \mu_{1}: V_{1}(\mathbb{G}) \rightarrow \Delta_{1}(\mathbb{G})$ is surjective. Indeed, given $g \in \Delta_{1}(\mathbb{G})$, there is a (unique) homomorphism $\varphi: \mathbb{G}_{U} \rightarrow \mathbb{G}$ such that $g=\varphi \circ g^{U}$, and we get $\mu_{1}\left(\varphi \circ f^{U}\right)=g$.

For constructing $f^{U}$ we will need a convenient "base immersion" in $\mathcal{A}$, the existence of which we now show.

Lemma 5.1. There exists $i_{0} \in \mathcal{A}$ whose image is contained in a ball $B \subseteq M$.

Proof. Let $i \in \mathcal{A}$ be some immersion and $H_{t}: F \rightarrow M$ a null-homotopy from $i$ to a constant map $k: F \rightarrow M$ with $k(F)=p \in B$. Using the parallelizability of $M$ we extend $H$ to a homotopy of bundle monomorphisms, from $d i$ to a bundle monomorphism $b$ which covers $k$. By the Smale-Hirsch Theorem applied to $\operatorname{Imm}(F, B)$, there is an immersion $i_{0}: F \rightarrow B$ with $d i_{0}$ homotopic to $b$ as bundle monomorphisms. Now by the Smale-Hirsch Theorem applied to $\operatorname{Imm}(F, M), i$ is regularly homotopic to $i_{0}$, and so $i_{0} \in \mathcal{A}$.

Fix an immersion $i_{0} \in I_{0}$ with image contained in a small ball $B \subseteq M$, as provided by Lemma 5.1. For any $i \in I_{0}$ take a generic regular homotopy $J_{t}$ from $i_{0}$ to $i$, and to each $\mathrm{CE}$ which occurs along $J_{t}$ choose a proper co-orientation. Let $f^{U}(i)$ be the sum of the elements in $\mathbb{G}^{U}$ corresponding to the co-oriented CEs occurring along $J_{t}$, each taken with a sign \pm according to whether $J_{t}$ passes it in the direction of its co-orientation. An opposite choice of co-orientation for a $\mathrm{CE}$ along $J_{t}$ (relevant for types $H, Q$ ) will produce the negative element in $\mathbb{G}_{U}$, but this element will appear with an opposite sign in the sum, and so this sum is well defined, for given $J_{t}$. We would like to show that in fact $f^{U}$ is independent of the regular homotopy $J_{t}$, and so $f^{U}$ is a well defined invariant. This is equivalent to showing that this sum is 0 along any closed $J_{t}$, i.e. $J_{t}$ such that $J_{0}=J_{1}=i_{0}$. Once we know $f^{U}$ is well defined, it is clear that it is of order 1 , and $\mu_{1}\left(f^{U}\right)=g^{U}$. 
The fact that the sum of values is 0 along any null-homotopic loop follows from the definition of $\Delta_{1}$ and $\mathbb{G}_{U}$ as demonstrated in [N3], and so the sum along loops induces a well defined homomorphism $\phi: \pi_{1}\left(\mathcal{A}, i_{0}\right) \rightarrow \mathbb{G}_{U}$. We must show $\phi=0$.

Fix a disc $D \subseteq F$, and let $S: \mathcal{A} \rightarrow \operatorname{Imm}(D, M)$ be given by restriction, $S(i)=$ $\left.i\right|_{D}$. Then $S$ is a fibration with fiber $\mathcal{A}_{D}=\left\{i \in \mathcal{A}:\left.i\right|_{D}=\left.i_{0}\right|_{D}\right\}$. So we get an exact sequence

$$
\pi_{1}\left(\mathcal{A}_{D}, i_{0}\right) \stackrel{i n c_{*}}{\longrightarrow} \pi_{1}\left(\mathcal{A}, i_{0}\right) \stackrel{S_{*}}{\longrightarrow} \pi_{1}\left(\operatorname{Imm}(D, M),\left.i_{0}\right|_{D}\right) .
$$

Let $K \subseteq \pi_{1}\left(\mathcal{A}, i_{0}\right)$ be the subgroup consisting of loops obtained by composing $i_{0}: F \rightarrow B$ with a motion of $B$ in $M$ through embeddings, beginning and ending with the inclusion of $B$ in $M$. It is clear that $S_{*}$ maps $K$ onto $\pi_{1}\left(\operatorname{Imm}(D, M),\left.i_{0}\right|_{D}\right)$, and so the elements of $K$ and those coming from $\pi_{1}\left(\mathcal{A}_{D}, i_{0}\right)$ generate $\pi_{1}\left(\mathcal{A}, i_{0}\right)$. Now $\phi$ is 0 on elements of $K$, since no CEs occur along such loops, and so it remains to show that $\phi$ is 0 on $\pi_{1}\left(\mathcal{A}_{D}, i_{0}\right)$. It is shown in the proof of [N1], Theorem 3.4, that $\pi_{1}\left(\mathcal{A}_{D}, i_{0}\right)$ is generated by a loop which only moves a small disc $U \subseteq F$ which is disjoint from $D$, and $U$ moves only within a small ball $B^{\prime}$. In our case we may take $B^{\prime} \subseteq B$, and so we finally need only to check the value of $\phi$ on loops moving $F$ in $B$. If we take our labeling to be locally constant above $B$, then we can have all lifts used to define our symbols to be contained in the same lift of $B$, constantly labeled

by some fixed element $h \in \pi_{a}$, and so all symbols will be of the form $R_{m h}^{h \epsilon_{1}, h \epsilon_{2}, \ldots}$. By a permutation of the superscript, which is even in case $R$ is $Q$, we may assume that all + signs appear first, and so the superscript is characterized only by the number of + signs, which is precisely the way the superscript of a symbol is defined in N3] for immersions $F \rightarrow B=\mathbb{R}^{3}$. The subscript is characterized by the coefficient $m$ of $m h$ which is also simply the degree as defined in [N3 for immersions $F \rightarrow B$. Therefore, $\phi$ being 0 on all loops moving $F$ in $B$ follows from the fact that $f^{U}$ appearing in [N3] for the case $M=\mathbb{R}^{3}$ is well defined. Indeed, for $f^{U}$ of [N3], the sum of symbols along a loop of immersions in $B$ is 0 in the group $\mathbb{G}_{U}$ of [N3]. We have noticed that our symbols of the form $R_{m h}^{h \epsilon_{1}, h \epsilon_{2}, \ldots}$ correspond to the symbols of [N3], but furthermore, any equation on the symbols appearing in [N3] has a corresponding equation on our symbols of the form $R_{m h}^{h \epsilon_{1}, h \epsilon_{2}, \ldots}$ by considering the same codimension 2 configuration in $B$. Therefore, the sum in our present $\mathbb{G}_{U}$ must also be 0 . This completes the proof of our main result, which classifies all order 1 invariants for any orientable aspherical 3-manifold:

Theorem 5.2. Let $F$ be a closed orientable surface, $M$ an aspherical orientable 3manifold, and $\mathcal{A}$ a regular homotopy class of null-homotopic immersions of $F$ into $M$. Then for any Abelian group $\mathbb{G}$, the injection $\mu_{1}: V_{1} / V_{0} \rightarrow \Delta_{1}$ is surjective.

\section{EXAMPLES}

Our classification of order 1 invariants (Theorem 5.2) is in terms of the group $\Delta_{1}(\mathbb{G})$ which as we have seen is the same as the group $\operatorname{Hom}\left(\mathbb{G}_{U}, \mathbb{G}\right)$. In [N3] where $M=\mathbb{R}^{3}$ and so $\pi_{1}(M)=\{1\}$, the structure of $\mathbb{G}_{U}$ is completely understood and accordingly the group of order 1 invariants. In the general case presented here, the structure of $\mathbb{G}_{U}$ depends on the structure of $\pi_{1}(M)$, most notably through the equivalence relation which produces $\mathcal{C}_{1}$ from $\mathcal{C}_{1}^{\prime}$. The complexity of this dependence may be seen in Examples 3.3 and 3.4 where the question is whether a given symbol is equivalent to its reversed symbol. Therefore, a closed explicit classification as appears in [N3] for $M=\mathbb{R}^{3}$ will require an extensive analysis of $\pi_{1}(M)$ for any 
given $M$. In this section we first find the set of all order 1 invariants which are common to all $M$. We then use one such invariant to prove a pair of equalities satisfied by any immersion in our setting. Finally, for $M$ with $\pi_{1}(M)=\mathbb{Z}^{k}, k \geq 1$, we demonstrate two large families of order 1 invariants that do not appear when $\pi_{1}(M)=0$.

We first ask, what is the set of invariants that are common to all orientable aspherical 3-manifolds. For this we must eliminate the role of $\pi_{1}(M)$. Given a symbol $R_{d}^{g_{1} \epsilon_{1}, \ldots, g_{|R|} \epsilon_{|R|}}$, we define its reduced symbol $R_{m}^{a}$, where $a$ is the number of + signs in $g_{1} \epsilon_{1}, \ldots, g_{|R|} \epsilon_{|R|}$ and $m$ is the sum of the coefficients in $d$. Accordingly, we define the reduced degree $d_{p}^{r}(i)$ of an immersion $i$ with respect to a point $p \in M$ as the sum of the coefficients of the degree in $\mathbb{Z}\left[\pi_{a}\right]$ defined in Section 3 . We define the reduced universal Abelian group $\mathbb{G}_{U}^{r}$ to be the quotient group of $\mathbb{G}_{U}$ with the added relations identifying any two symbols if they correspond to the same reduced symbol. The group $\mathbb{G}_{U}^{r}$ is the same group for all $M$, and it is equal to $\mathbb{G}_{U}$ itself when $\pi_{1}(M)=\{1\}$; i.e. it is the group $\mathbb{G}_{U}$ of $\mathbb{R}^{3}$ appearing in [N3. If $\rho: \mathbb{G}_{U} \rightarrow \mathbb{G}_{U}^{r}$ is the natural map, then $\rho \circ f^{U}$ is an order one invariant which is common to all 3 -manifolds $M$, which in the case $\pi_{1}(M)=\{1\}$ is the universal order 1 invariant itself. The conclusion is that all order 1 invariants existing in $\mathbb{R}^{3}$ and thoroughly studied in [N3], [N5] exist in all $M$. More precisely, let $\mathbb{G}_{U}^{M}, f_{M}^{U}$ be the universal group and universal order 1 invariant for $M$. Then any order 1 invariant on $\mathbb{R}^{3}$ is given up to a constant as $\varphi \circ f_{\mathbb{R}^{3}}^{U}$ for some homomorphism $\varphi: \mathbb{G}_{U}^{\mathbb{R}^{3}} \rightarrow \mathbb{G}$, and the corresponding invariant on $M$ will be $\varphi \circ \rho \circ f_{M}^{U}$. The invariant $f_{\mathbb{R}^{3}}^{U}$ is presented in detail in N5], Section 3, and the geometric meaning of each of its components is explained. The same geometric meaning applies to the components of $\rho \circ f_{M}^{U}$ for general $M$.

We give an example of one explicit order 1 invariant appearing in [N5] with an application. We will not present the invariant in terms of such $\varphi$ but rather give an explicit formula for it presented in [N5]. This explicit formula applies to any $M$ for which the Euler characteristic of the subsets appearing below is always finite, so assume $M$ may be presented as the interior of a compact manifold (which includes the case where $M$ is closed).

Let $i \in I_{0}$. For every $m \in \mathbb{Z}$ let $U_{m}=U_{m}(i)=\left\{p \in M-i(F): d_{p}^{r}(i)=\right.$ $m\}$. Denote by $N_{m}=N_{m}(i)$ the number of triple points $p \in M$ of $i$ having $d_{p}^{r}(i)=m$. We define the group $\mathbb{O}$ to be the free Abelian group with generators $\left\{x_{n}\right\}_{n \in \mathbb{Z}} \cup\left\{y_{n}\right\}_{n \in \mathbb{Z}}$. For $i \in I_{0}$ we define $k(i) \in \mathbb{O}$ as follows:

$$
k(i)=\sum_{m \in \mathbb{Z}} \chi\left(U_{m}\right) x_{m}+\sum_{m \in \mathbb{Z}} \frac{1}{2} N_{m} y_{m} .
$$

The same proof as in N5 shows that this is indeed an order 1 invariant. In this case, to show that the coefficients appearing in the formula are indeed integers (and never half integers), we must again show that there is some immersion in any regular homotopy class for which the coefficients are integers. By Lemma 5.1 above, there exists $i \in \mathcal{A}$ whose image is contained in a ball $B$. We may continue by regular homotopy in $B$ to an immersion $j$ as appearing in [N5], Lemma 5.2. This $j$ in a general $M$ gives $k(j)=(\chi(M)+1-g) x_{0}+(1-g) x_{-1}$, where $g$ is the genus of $F$.

We present an application of the invariant $k$. As in $\left[\mathbf{N 5}\right.$, let $\theta_{0}: \mathbb{O} \rightarrow \mathbb{Z}$ be the homomorphisms defined by $\theta_{0}\left(x_{2 m}\right)=1, \theta_{0}\left(x_{2 m+1}\right)=0, \theta_{0}\left(y_{m}\right)=-1$ for all $m$ and $\theta_{1}: \mathbb{O} \rightarrow \mathbb{Z}$ defined by $\theta_{1}\left(x_{2 m}\right)=0, \theta_{1}\left(x_{2 m+1}\right)=1, \theta_{1}\left(y_{m}\right)=-1$ for all $m$. 
Then as in N5 we have that $\theta_{0} \circ k$ and $\theta_{1} \circ k$ are constant invariants, which are given explicitly by $\theta_{0} \circ k(i)=\sum \chi\left(U_{2 m}\right)-\frac{1}{2} N$ and $\theta_{1} \circ k(i)=\sum \chi\left(U_{2 m+1}\right)-\frac{1}{2} N$, where $N=N(i)=\sum N_{m}(i)$ is the total number of triple points of $i$. To find the value of these constants we need to evaluate them on a single immersion in every regular homotopy class. For the immersion $j$ above, $\theta_{0} \circ k(j)=\chi(M)+1-g$ and $\theta_{1} \circ k(j)=1-g$, so we get the following two identities:

Proposition 6.1. Let $g$ be the genus of $F$. For any $i \in I_{0}$,

$$
\sum_{m} \chi\left(U_{2 m}\right)-\frac{1}{2} N=\chi(M)+1-g \quad \text { and } \quad \sum_{m} \chi\left(U_{2 m+1}\right)-\frac{1}{2} N=1-g .
$$

Note that this pair of equalities is finer than what can be deduced from the result of Izumiya and Marar in [IM], which for immersions is $\chi(i(F))=\chi(F)+N$. Indeed, think of $M$ as the union of a regular neighborhood of $i(F)$, and a slightly diminished $\bigcup_{m} U_{m}$. The intersection of the two is $\partial \bigcup_{m} U_{m}-\partial M$ (recall we assume for our formula that $M$ is the interior of a compact manifold, so we include the boundary here if it exists). We get $\chi(M)=\chi(i(F))+\sum \chi\left(U_{m}\right)-\sum \chi\left(\partial U_{m}\right)+\chi(\partial M)$. Now using the fact that for any compact 3-manifold $L, \chi(\partial L)=2 \chi(L)$, we get $\chi(i(F))=$ $\sum \chi\left(U_{m}\right)-\chi(M)$. This shows that the Izumiya Marar equality is equivalent to $\sum \chi\left(U_{m}\right)-N=\chi(M)+\chi(F)$, which is the sum of our two equalities.

Finally, we look at $M$ so that $\pi_{1}(M)$ is a finitely generated Abelian group. By standard considerations concerning group homology, the only finitely generated Abelian groups that may appear as the fundamental group of an aspherical 3manifold are $\mathbb{Z}^{k}, 0 \leq k \leq 3$. For $k>0$ we will now construct two large families of order 1 invariants that do not appear for $k=0$. (These groups are indeed realized by orientable aspherical 3-manifolds, namely, $S^{1} \times \mathbb{R}^{2}, S^{1} \times S^{1} \times \mathbb{R}$ and $S^{1} \times S^{1} \times S^{1}$.) Choose an ordering $\preceq$ on $\mathbb{Z}^{k}$ which is invariant under the action of $\mathbb{Z}^{k}$ on itself, e.g. the lexicographical ordering induced by the usual ordering of $\mathbb{Z}$. Let $A=\left\{v \in \mathbb{Z}^{k}: 0 \preceq v\right\}$; then any symbol of the form $H_{d}^{g_{1} \epsilon_{1}, g_{2} \epsilon_{2}}$ is equivalent to a symbol of the form $H_{d}^{0 \epsilon_{1}, v \epsilon_{2}}$ for unique $v \in A$, and the same is true for $E_{d}^{g_{1} \epsilon_{1}, g_{2} \epsilon_{2}}$. Now let $h: A \rightarrow \mathbb{G}$ be an arbitrary function. We will use $h$ to define an order 1 invariant $f$. We first define $g \in \mathcal{C}_{n}^{*}$ as follows: For symbol $x$ of the form $H_{d}^{g_{1}+, g_{2}+}$, let $g(x)=h(v)$ for the unique $v$ mentioned above. For $x$ of the form $H_{d}^{g_{1}-, g_{2}-}$ let $g(x)=-h(v)$, and for $x$ of the form $H_{d}^{g_{1}+, g_{2}-}$ let $g(x)=0$. For any $x$ of the form $E_{d}^{g_{1} \epsilon_{1}, g_{2} \epsilon_{2}}$, define $g(x)$ in the same way. Finally, for any symbol $x$ of configuration $T$ or $Q$ define $g(x)=0$. One can easily check that $g$ is well defined on equivalence classes of symbols and satisfies all eight relations defining $\Delta_{1}$, and so by Theorem 5.2 there is an order 1 invariant $f$ with values in $\mathbb{G}$ such that $\mu_{1}(f)=g$. So, for any choice of function $h: A \rightarrow \mathbb{G}$ we have constructed an order 1 invariant $f$, and note that $f$ belongs to the set of invariants which are common to all $M$ only if $h$ is a constant function.

We now define a second such family of invariants. Let $B=\left\{(v, w) \in \mathbb{Z}^{k} \times\right.$ $\left.\mathbb{Z}^{k}: 0 \preceq v \preceq w\right\}$; then any symbol of the form $T_{d}^{g_{1} \epsilon_{1}, g_{2} \epsilon_{2}, g_{3} \epsilon_{3}}$ is equivalent to a symbol of the form $T_{d}^{0 \epsilon_{1}, v \epsilon_{2}, w \epsilon_{3}}$ for unique $(v, w) \in B$. Now let $h: B \rightarrow \mathbb{G}$ be an arbitrary function. We use it to define $g \in \mathcal{C}_{n}^{*}$ as follows: For symbol $x$ of the form $T_{d}^{g_{1} \epsilon_{1}, g_{2} \epsilon_{2}, g_{3} \epsilon_{3}}$ let $g(x)=h(v, w)$ for the unique $(v, w)$ mentioned, and, for any symbol $x$ of configuration $E, H$ or $Q$ define $g(x)=0$. Again, one can easily check that $g$ is well defined on equivalence classes of symbols and satisfies all eight relations defining $\Delta_{1}$, and so there is an order 1 invariant $f$ such that $\mu_{1}(f)=g$. 
Again, $f$ belongs to the set of invariants which are common to all $M$ only if $h$ is a constant function.

\section{REFERENCES}

[HK] C. A. Hobbs, N. P. Kirk, "On the Classification and Bifurcation of Multigerms of Maps from Surfaces to 3-Space." Mathematica Scandinavica 89 (2001) 57-96. MR 1856982 (2002g:58069)

[IM] S. Izumiya, W. Marar, "The Euler Characteristic of a Generic Wavefront in a 3-Manifold." Proceedings of the American Mathematical Society 118 (1993) 1347-1350. MR1165055 (93j:58014)

[N1] T. Nowik, "Quadruple points of regular homotopies of surfaces in 3-manifolds." Topology 39 (2000) 1069-1088. MR.1763964 (2001i:57033)

[N2] T. Nowik, "Automorphisms and Embeddings of Surfaces and Quadruple Points of Regular Homotopies." Journal of Differential Geometry 58 (2001) 421-455. MR1906781 (2003i:57034)

[N3] T. Nowik, "Order one invariants of immersions of surfaces into 3-space." Mathematische Annalen 328 (2004) 261-283. MR2030377 (2004m:57025)

[N4] T. Nowik, "Higher order invariants of immersions of surfaces into 3-space." Pacific Journal of Mathematics 223 (2006) 333-347. MR2221030 (2007b:57024)

[N5] T. Nowik, "Formulae for Order One Invariants of Immersions of Surfaces." Advances in Mathematics 206 (2006), no. 2, 358-372. MR2263707 (2007j:57031)

[N6] T. Nowik, "Immersions of Non-orientable Surfaces." Topology Appl. 154 (2007), no. 9, 18811893. MR2319260 (2008b:57032)

Department of Mathematics, Bar-Ilan University, Ramat-Gan 52900, Israel

E-mail address: tahl@math.biu.ac.il

URL: http://www. math.biu.ac.il/ $\sim \operatorname{tahl}$ 